\title{
Conhecimento sobre o tratamento farmacológico em pacientes com doença renal crônica
}

\author{
Leonardo Barbosa Moreira', Paula Frassinetti Castelo Branco Camurça Fernandes², Francisca \\ Sueli Monte ${ }^{1}$, Rita Izabel Monteiro Galvão ${ }^{2}$, Alice Maria Costa Martins ${ }^{1 *}$
}

\author{
${ }^{1}$ Faculdade de Farmácia, Odontologia e Enfermagem, Universidade Federal do Ceará, ${ }^{2}$ Faculdade de Medicina, \\ Universidade Federal do Ceará
}

\footnotetext{
*Correspondência:

A. M. C. Martins

Departamento de Análises Clínicas e Toxicológicas

Faculdade de Farmácia, Odontologia

e Enfermagem

Universidade Federal do Ceará

Rua Cap. Francisco Pedro, 1210,

bairro Rodolfo Teófilo

CEP: 60430-170 - Fortaleza - CE, Brasil

E-mail: martinsalice@gmail.com
}

O adequado conhecimento dos pacientes sobre os medicamentos que utilizam é considerado um fator fundamental para a adesão ao tratamento. O nivel de conhecimento sobre a terapia farmacológica de pacientes com doença renal crônica (DRC) e os fatores associados a este conhecimento foram avaliados em um estudo transversal envolvendo 130 individuos em uso contínuo de algum medicamento pertencente aos grupos C, H02 ou LO4 da classificação Anatomical Therapeutic Chemical e que não estivessem sendo submetidos a qualquer terapia de substituição renal. O nivel de conhecimento foi mensurado através de um questionário e um escore de zero a dez pontos. Foram estabelecidos três niveis de conhecimento: baixo (menos de seis pontos), médio (seis a oito pontos) e bom (mais de oito pontos). O escore médio foi de 7,8 $\pm 1,7$ pontos e $51,5 \%$ dos pacientes apresentaram nivel de conhecimento baixo ou médio. Os fatores associados ao alto nivel de conhecimento foram: declínio rápido do ritmo de filtração glomerular, controle da pressão arterial e crença do paciente em já ter sofrido reação adversa a algum medicamento prescrito. A maioria dos entrevistados possuía conhecimentos insuficientes para o uso seguro e eficaz dos medicamentos, o que sugere a necessidade de melhor orientação aos pacientes.

\section{INTRODUÇÃO}

A doença renal crônica (DRC) representa, atualmente, um importante problema de saúde pública no Brasil (Romão Júnior, 2004). Quase um milhão de brasileiros sofrem de problemas renais e $70 \%$ não são diagnosticados. As doenças renais levam ao óbito no mínimo 15 mil pessoas por ano no país.
Os custos da atenção aos pacientes portadores de nefropatias são de $\mathrm{R} \$ 1,4$ bilhão por ano, o que representa $10 \%$ de toda a verba destinada à saúde. Cerca de 60 mil pessoas fazem diálise no Brasil e 25 mil iniciam o tratamento dialítico por ano, entretanto, pelo menos 150 mil deveriam ser submetidos ao tratamento. Somente três mil pacientes recebem um transplante renal por ano e 25 mil transplantados estão em acompanha- 
mento. Trinta e quatro milhões de brasileiros sofrem de diabetes ou hipertensão e 6,6\% destes são doentes renais (Sociedade Brasileira de Nefrologia, 2006).

Um dos objetivos mais importantes do tratamento da DRC é retardar a progressão da enfermidade, através do tratamento específico das doenças de base que constituem o seu diagnóstico etiológico (National Kidney Foundation, 2002; Romão Júnior, 2004). No Brasil o diabetes melito e a hipertensão arterial sistêmica são doenças de base freqüentes entre os pacientes com DRC (Romão Júnior, 2004). Estas condições podem ser devidamente controladas através do uso de fármacos seguros e eficazes (Chobanian et al., 2003; American Diabetes Association, 2006). Entretanto, a não adesão à terapia farmacológica permanece como uma importante barreira à obtenção dos resultados ótimos no tratamento da hipertensão e do diabetes (Lee et al., 1996; Grant et al., 2003; Kuo et al., 2003; Strelec, Pierin, Mion Júnior, 2003; Kilbourne et al., 2005).

A perda da cooperação por parte do paciente freqüentemente significa a falha da terapia, acarretando sofrimento desnecessário, hospitalizações ou mesmo a morte precoce (Bunzel, Laederach-Hofmann, 2000; Cramer, 2002). Nos Estados Unidos cerca de 5,3\% das admissões hospitalares estão associadas à não adesão aos tratamentos farmacológicos prescritos, enquanto os custos anuais diretos e indiretos decorrentes deste comportamento são de 25 bilhões e 100 bilhões de dólares, respectivamente. Estas estimativas incluem os custos com medicamentos não utilizados, consultas adicionais, atendimentos de emergência, hospitalizações e testes diagnósticos (Teixeira et al., 2000; Rybacki, 2002).

Diversos fatores associados ao paciente, à doença, ao tratamento, aos profissionais e aos serviços de saúde parecem ter influência sobre o comportamento aderente ou não aderente (Sarquis et al., 1998; Leite, Vasconcellos, 2003; World Health Organization, 2003). Entre estes fatores está o adequado nível de conhecimento dos pacientes sobre o tratamento medicamentoso, considerado um fator essencial para a utilização dos medicamentos conforme a prescrição médica (Farley, 1995; Okuno et al., 1999; Bunzel, Laederach-Hofmann, 2000; Silva, Schenkel, Mengue, 2000; Sano et al., 2002; Vasquez et al., 2003; Burge et al., 2005).

Diante do exposto, realizamos um estudo com o objetivo de mensurar o nível de conhecimento sobre o tratamento farmacológico em pacientes com doença renal crônica e identificar os fatores que estão associados a este conhecimento.

\section{MATERIAL E MÉTODOS}

O estudo seguiu o delineamento transversal e foi realizado no ambulatório de nefrologia do Hospital Univer- sitário Walter Cantídio/Universidade Federal do Ceará (HUWC/UFC), um hospital geral, universitário, público e de nível terciário, localizado na cidade de Fortaleza (CE). A coleta dos dados ocorreu no período de novembro de 2004 a maio de 2005 .

Foram incluídos pacientes maiores de 18 anos com DRC; em uso contínuo de pelo menos um medicamento pertencente aos grupos $\mathrm{C}$ (medicamentos que atuam no sistema cardiovascular), H02 (corticoesteróides de uso sistêmico) ou L04 (medicamentos imunossupressores) da classificação Anatomical Therapeutic Chemical (ATC), comprovado através de apresentação da prescrição do dia; que não estivessem sendo submetidos a diálise peritoneal, hemodiálise ou transplante renal; e que compareceram a pelo menos uma consulta durante o período do estudo. Foram seguidos os critérios de diagnóstico e estadiamento da DRC estabelecidos pela Sociedade Brasileira de Nefrologia (SBN), que considera os pacientes com hipertensão, diabetes melito e doenças glomerulares primárias ou secundárias doentes renais crônicos de estágio 0 (Romão Júnior, 2004). A classificação $A T C$ foi escolhida por ser a recomendada pela Organização Mundial da Saúde (World Health Organization, 2005). Para o recrutamento da amostra foi adotado o método de amostragem não probabilístico consecutivo. Desta forma, foram recrutados consecutivamente todos os pacientes que atendiam aos critérios de inclusão durante o período do estudo.

Os pacientes recrutados foram entrevistados após a consulta médica no ambulatório de nefrologia utilizandose um questionário semi-estruturado aplicado pelo pesquisador. O instrumento foi adaptado a partir do modelo desenvolvido por Silva (1999). As perguntas referiam-se ao nome, indicação, dose, freqüência de administração, intervalo de doses, duração do tratamento e possíveis reações adversas de cada um dos medicamentos pertencentes aos grupos $\mathrm{C}, \mathrm{H} 02$ ou L04 da classificação $A T C$, de uso contínuo pelo paciente e contidos na prescrição do dia. O questionário também serviu para coletar dados sobre as percepções dos pacientes sobre a doença e o tratamento farmacológico, além de dados sociodemográficos. Foram realizadas consultas aos prontuários médicos empregandose um formulário semi-estruturado para coletar dados sobre a doença, os medicamentos em uso, aferições de pressão arterial e resultados de dosagens laboratoriais de creatinina sérica e proteinúria de 24 horas realizadas nos 12 meses anteriores à entrevista. O questionário e o formulário de coleta de dados do prontuário foram previamente testados em um estudo piloto.

A coleta de dados foi realizada por um único pesquisador em três etapas: na primeira etapa foram realizadas buscas nos prontuários dos pacientes que tinham consulta 
agendada e elaborada uma relação de pacientes elegíveis. $\mathrm{Na}$ segunda etapa, que ocorreu na sala de espera do ambulatório, foram realizadas as entrevistas. Primeiramente era feita a verificação dos pacientes que compareceram à consulta e em seguida era realizada a abordagem. Neste momento era averiguado se o paciente era responsável pelo seu próprio tratamento e, nos casos positivos, era solicitada a sua participação no estudo. Foi explicado aos pacientes que se tratava de uma pesquisa sobre como as pessoas que têm "problemas de rins" estão usando os medicamentos receitados e que estas informações seriam úteis para melhorar os seus respectivos tratamentos. Foi solicitado aos pacientes que aceitaram participar da pesquisa que, logo após a consulta, permanecessem na sala de espera para a realização da entrevista. Quando os pacientes saíam do consultório, era observado se eles haviam recebido a prescrição médica contendo algum medicamento de interesse e, nos casos positivos, o paciente era recrutado para somente então ser iniciada a entrevista. Durante a entrevista as perguntas foram lidas para todos os pacientes de forma padronizada e foi permitido a eles consultar a prescrição ou qualquer outro material informativo para respondê-las. Ao final de cada entrevista a prescrição médica era transcrita pelo entrevistador. As entrevistas duravam entre 10 e 20 minutos dependendo do número de medicamentos incluídos. Durante a terceira etapa da coleta dos dados foram realizadas consultas aos prontuários dos pacientes entrevistados.

Para a análise das respostas dos pacientes sobre os medicamentos foram estabelecidos critérios de julgamento que definiam se elas estavam corretas ou não. Deveria haver concordância entre as respostas dos pacientes e as informações contidas nas fontes de referência, mesmo que os pacientes não utilizassem os termos técnicos corretos. A fonte de informação de referência para o julgamento das respostas sobre o nome, dose, freqüência de administração e intervalo de doses foi a prescrição médica; para a indicação terapêutica e a duração do tratamento a referência foi o prontuário médico e para as possíveis reações adversas foi o livro Martindale: The Complete Drug Reference $32^{\text {th }}$ ed. (Parfitt, 1999). Para os nomes dos medicamentos foram aceitas respostas em nomes comerciais ou genéricos. A análise das respostas foi feita de forma independente por dois revisores. Deveria haver concordância de parecer entre ambos. Em caso de discordância foi consultado um terceiro revisor, que decidiu sobre a questão. Dos revisores, dois eram farmacêuticos e um era médico.

Com base no julgamento das respostas dos pacientes foi empregado um escore para quantificar o nível de conhecimento que eles possuíam sobre o tratamento farmacológico prescrito, também adaptado de Silva (1999). Para cada resposta correta foram atribuídos ao entrevistado dois pontos para o nome, dois pontos para a dose, dois pontos para a freqüência, um ponto para a indicação, um ponto para o intervalo de doses, um ponto para a duração do tratamento e um ponto para efeitos adversos, totalizando o máximo de dez pontos por medicamento em uso. Para cada resposta considerada incorreta pelos revisores não foi atribuída pontuação alguma para o quesito. Quando o paciente utilizava mais de um medicamento o seu escore final foi o resultado da média aritmética dos escores de cada um.

$\mathrm{O}$ adequado conhecimento sobre todos os aspectos avaliados é importante para o uso seguro dos medicamentos no contexto ambulatorial, entendido como o uso que minimize a ocorrência de danos à saúde e ao bem estar do paciente. Entretanto, os quesitos nome, dose e freqüência de utilização foram considerados essenciais para a correta aquisição e administração dos medicamentos e, portanto receberam peso dois. A partir da pontuação média alcançada pelo paciente ele era classificado em um dos três níveis de conhecimento: baixo (menos de seis pontos), quando o uso dos medicamentos foi considerado inseguro; médio (seis a oito pontos), quando o uso dos medicamentos foi considerado seguro desde que não haja intercorrências durante o tratamento e alto (mais de oito pontos) quando o uso dos medicamentos foi considerado seguro sob todas as circunstâncias (Silva, Schenkel, Mengue, 2000).

Os parâmetros clínicos foram analisados de acordo com as metas terapêuticas estabelecidas pela SBN (Alves, 2004; Bregman, 2004). Desta forma foram considerados pacientes com declínio rápido no ritmo de filtração glomerular (RFG), estimado a partir da creatinina sérica ajustada pela equação de Cockcroft-Gault (Pecoits-Filho, 2004), aqueles que apresentavam diminuição maior do que $4 \mathrm{~mL} / \mathrm{min} / 1,73 \mathrm{~m}^{2} /$ ano. Pacientes que apresentavam pressão arterial sistólica média maior que $135 \mathrm{mmHg}$, ou pressão arterial diastólica média maior que $85 \mathrm{mmHg}$, quando a proteinúria média fosse menor que $1 \mathrm{~g} / 24 \mathrm{~h}$; ou pressão arterial sistólica média maior que $125 \mathrm{mmHg}$, ou pressão arterial diastólica média maior que $75 \mathrm{mmHg}$, quando a proteinúria média fosse maior que $1 \mathrm{~g} / 24 \mathrm{~h}$ foram considerados não controlados.

Para o dimensionamento da amostra foi empregado o conjunto de programas Epi Info 6, versão 6.04d, 2001 (Centers for Disease Control and Prevention, Atlanta, GA, USA), admitindo-se o nível de significância de $95 \%$ e o poder do estudo de $80 \%$. A partir de um estudo piloto foram estimadas as prevalências utilizadas no cálculo, que resultou em uma amostra de 128 pacientes. O Epi Info 6 também foi utilizado na análise dos dados do estudo para a realização da estatística descritiva, utilizando-se medidas de tendência central e dispersão (média \pm desvio padrão), além das distribuições de freqüências, e para a análise bivariada, 
utilizando-se o teste do Qui-quadrado corrigido por Yates. As variáveis que apresentaram associação estatística com o alto nível de conhecimento sobre o tratamento farmacológico com nível de significância menor que 0,20 na análise bivariada foram incluídas em um modelo de regressão logística múltipla para a avaliação dos efeitos no contexto multifatorial, utilizando-se o programa SPSS for Windows, versão 11.5 (Chicago, IL, USA). Em todos os testes estatísticos as diferenças foram consideradas estatisticamente significantes para " $p$ " menor que 0,05 (bicaudal).

O estudo foi aprovado pelo Comitê de Ética em Pesquisa da Universidade Federal do Ceará. Foi solicitado o consentimento escrito a cada participante do estudo. O Termo de Consentimento Livre e Esclarecido para Participação em Pesquisa era lido para o paciente e em seguida era solicitada a sua assinatura. Foi garantido o sigilo sobre as respostas e respeitada a recusa do paciente em responder a qualquer pergunta do questionário.

\section{RESULTADOS}

Havia 205 pacientes elegíveis com consultas médicas agendadas durante o período do estudo. Deste total, 23 pacientes $(11,2 \%)$ não compareceram à consulta; 23 pacientes $(11,2 \%)$ não receberam prescrição médica contendo medicamentos pertencentes aos grupos C, H02 ou L04 da classificação $A T C$ dos quais estivessem em uso contínuo; 17 pacientes $(8,3 \%)$ recusaram-se a participar do estudo e 12 pacientes $(5,9 \%)$ não eram os responsáveis pela administração dos próprios medicamentos e requeriam a ajuda de um cuidador. Os 130 pacientes restantes foram recrutados para o estudo.

As características sociodemográficas, características e percepções sobre a doença renal crônica e características e percepções sobre o tratamento farmacológico observadas na amostra são apresentadas na Tabela I. A maior parte dos entrevistados era do sexo feminino (73,1\%). Aidade média foi de 48,8 $\pm 15,8$ anos (18 a 79 anos). Houve predomínio de pacientes analfabetos ou que tinham como escolaridade apenas o ensino fundamental (68,5\%). O lupus eritematoso sistêmico (LES) foi a doença de base mais prevalente entre os entrevistados $(29,3 \%)$, uma vez que o estudo foi realizado em um hospital universitário de nível terciário. Os diagnósticos de hipertensão arterial primária foram validados por um nefrologista segundo os critérios estabelecidos por Frassinetti Fernandes (2000). O tempo médio de diagnóstico de DRC foi de $8,1 \pm 6,2$ anos (0,2 a 21,7 anos) e o tempo médio de acompanhamento pelo ambulatório de nefrologia foi de 5,8 $\pm 4,8$ anos ( 0,1 a 20,1 anos). A maioria dos pacientes $(54,8 \%)$ estava com DRC nos estágios 3 , 4 ou 5 . Seis apresentavam RFG entre 10 e $15 \mathrm{~mL} / \mathrm{min} / 1,73$ $\mathrm{m}^{2}$, entretanto não estavam sendo submetidos a nenhuma terapia de substituição renal (TSR) ainda, pois estavam assintomáticos. Estes pacientes estavam aguardando a confecção da fístula artério-venosa e em seguida iniciariam a TSR. O declínio médio do RFG foi de $10,5 \pm 27,2 \mathrm{~mL} / \mathrm{min} /$ $1,73 \mathrm{~m}^{2} /$ ano (0 a $219 \mathrm{~mL} / \mathrm{min} / 1,73 \mathrm{~m}^{2} /$ ano), embora $63,0 \%$ dos indivíduos tivessem declínio normal $(\leq 4 \mathrm{~mL} / \mathrm{min} / 1,73$ $\mathrm{m}^{2} / \mathrm{ano}$ ). Apenas 31 entrevistados estavam com a pressão arterial adequadamente controlada enquanto $68,5 \%$ não sabiam do risco de serem submetidos à TSR em caso de não utilização dos medicamentos conforme prescrito pelo médico. O número total de medicamentos prescritos para uso no mês anterior à entrevista foi, em média, de 3,7 $\pm 1,6$ medicamentos por paciente ( 1 a 9 medicamentos por paciente). De um total de 74 medicamentos observados, o mais freqüente foi o captopril, prescrito a 55 indivíduos $(42,3 \%)$. O tempo médio de utilização dos medicamentos foi de 4,4 $\pm 3,9$ anos (0,1 a 20,3 anos). Aproximadamente $36 \%$ dos entrevistados acreditavam já ter sofrido reação adversa a algum medicamento prescrito.

A Tabela II apresenta os índices de acerto das respostas dos pacientes às perguntas sobre o tratamento farmacológico. Para as características nomes dos medicamentos, doses e freqüências de administrações os índices de acerto total, ou seja, quando houve acerto das respostas para todos os medicamentos em uso pelo paciente, foram de $77,7,90,0$ e $86,1 \%$, respectivamente.

O escore médio do nível de conhecimento sobre o tratamento farmacológico foi de $7,8 \pm 1,7$ pontos ( 1 a 10 pontos). A categoria mais freqüente foi a dos pacientes com alto nível de conhecimento (48,5\%; IC95\% = 39,6 $57,4 \%$ ), seguido daqueles com nível médio (41,5\%; IC95\% $=33,0-50,5 \%)$ e baixo $(10,0 \%$; IC95\% $=5,4-16,5 \%)$.

As características que apresentaram associação com o alto nível de conhecimento sobre o tratamento farmacológico, com nível de significância menor que 0,20 na análise bivariada, são demonstradas na Tabela III. Os fatores significativamente associados com maior conhecimento sobre os medicamentos foram: sexo feminino; idade até 50 anos; não ser chefe de família; escolaridade maior que o ensino fundamental; diagnóstico de DRC há pelo menos 5 anos; declínio rápido do RFG; utilizar algum medicamento pertencente aos grupos $\mathrm{H} 02$ ou L04 da classificação $A T C$; utilizar os medicamentos, em média, há mais de 3 anos; e acreditar já ter sofrido reação adversa a algum medicamento prescrito.

Após a utilização do modelo de regressão logística múltipla as características que apresentaram associação estatisticamente significante com o alto nível de conhecimento sobre o tratamento farmacológico foram: o declínio rápido do RFG (razão de chances ajustada $=3,33$; IC95\% 
TABELA I - Distribuição dos pacientes incluídos na amostra, segundo as características gerais avaliadas $(\mathrm{n}=130)$

\begin{tabular}{|c|c|c|}
\hline Características & $\mathrm{n}$ & $\%$ \\
\hline Sexo feminino & 95 & 73,1 \\
\hline Idade $\leq 50$ anos & 68 & 52,3 \\
\hline Vive com a família & 126 & 96,9 \\
\hline Chefe de família & 50 & $39,7^{1}$ \\
\hline Escolaridade máxima ensino fundamental completo & 89 & 68,5 \\
\hline Exerce atividade remunerada & 29 & 22,3 \\
\hline Renda familiar total $\leq 2$ salários mínimos ${ }^{2}$ & 74 & $62,2^{3}$ \\
\hline \multicolumn{3}{|l|}{ Doença de base } \\
\hline Lupus eritematoso sistêmico & 36 & 29,3 \\
\hline Diabetes melito & 25 & 20,3 \\
\hline Doenças glomerulares primárias & 19 & 15,4 \\
\hline Hipertensão arterial primária & 19 & $15,4^{4}$ \\
\hline Tempo de diagnóstico $\geq 5$ anos & 81 & 62,3 \\
\hline Tempo de acompanhamento pelo ambulatório de nefrologia $\leq 5$ anos & 67 & 51,5 \\
\hline Estágio 3 a 5 da doença renal crônica & 68 & $54,8^{5}$ \\
\hline Declínio rápido do ritmo de filtração glomerular & 40 & $37,0^{6}$ \\
\hline Pressão arterial controlada & 31 & 23,8 \\
\hline $\begin{array}{l}\text { Desconhece o risco de ser submetido à terapia de substituição renal em } \\
\text { caso de não utilização dos medicamentos conforme prescrito }\end{array}$ & 89 & 68,5 \\
\hline Número de medicamentos prescritos $\geq 4$ & 70 & 53,9 \\
\hline \multicolumn{3}{|l|}{ Medicamentos mais freqüentes (código Anatomical Therapeutic Chemical) } \\
\hline Captopril (C09AA01) & 55 & 42,3 \\
\hline Hidroclorotiazida (C03AA03) & 44 & 33,8 \\
\hline Furosemida (C03CA01) & 41 & 31,5 \\
\hline Prednisona (H02AB07) & 41 & 31,5 \\
\hline Tempo médio de utilização dos medicamentos $\leq 3$ anos & 67 & 51,5 \\
\hline Acredita já ter sofrido reação adversa a algum medicamento prescrito & 46 & $35,9^{7}$ \\
\hline
\end{tabular}

${ }^{1}$ Quatro pacientes informaram que não viviam com suas famílias. ${ }^{2} \mathrm{O}$ salário mínimo vigente à época do estudo era de $\mathrm{R} \$$ $260,00 .{ }^{3}$ Onze pacientes não informaram a renda familiar. ${ }^{4}$ Sete pacientes não apresentavam o diagnóstico etiológico em seus prontuários. ${ }^{5}$ Seis pacientes tinham dados insuficientes em seus prontuários para o estadiamento da doença. ${ }^{6}$ Vinte e dois pacientes tinham dados insuficientes em seus prontuários para a estimativa da taxa de declínio do ritmo de filtração glomerular. ${ }^{7}$ Dois pacientes não responderam à pergunta sobre reação adversa aos medicamentos.

TABELA II - Distribuição dos pacientes incluídos na amostra, segundo os índices de acerto das respostas às perguntas sobre o tratamento farmacológico $(\mathrm{n}=130)$

\begin{tabular}{lcccccc}
\hline Característica & \multicolumn{2}{c}{ Sem acerto } & \multicolumn{2}{c}{ Acerto parcial } & \multicolumn{2}{c}{ Acerto total } \\
& $\mathrm{n}$ & $\%$ & $\mathrm{n}$ & $\%$ & $\mathrm{n}$ & $\%$ \\
\hline Nomes dos medicamentos & 13 & 10,0 & 16 & 12,3 & 101 & 77,7 \\
Indicações terapêuticas & 13 & 10,0 & 19 & 14,6 & 98 & 75,4 \\
Doses & 4 & 3,1 & 9 & 6,9 & 117 & 90,0 \\
Freqüências das administrações & 7 & 5,4 & 11 & 8,5 & 112 & 86,1 \\
Intervalos das doses & 19 & 14,6 & 30 & 23,1 & 81 & 62,3 \\
Durações dos tratamentos & 50 & 38,5 & 5 & 3,8 & 75 & 57,7 \\
Reações adversas & 78 & 60,0 & 32 & 24,6 & 20 & 15,4 \\
\hline
\end{tabular}


$=1,24-8,96 ; \mathrm{p}=0,017)$, o controle da pressão arterial (razão de chances ajustada $=4,87$;C95\% $=1,60-14,77$; $\mathrm{p}=0,005)$ e o fato do paciente acreditar já ter sofrido reação adversa a algum medicamento prescrito (razão de chances ajustada $=15,57 ;$ IC95\% $=4,96-48,90 ; \mathrm{p}<$ 0,001) (Tabela IV).

\section{DISCUSSÃO}

O adequado nível de conhecimento dos pacientes sobre os medicamentos que utilizam é considerado um fator essencial para a adesão ao tratamento farmacológico (Farley, 1995; Okuno et al., 1999; Bunzel, Laederach-

TABELA III - Características dos pacientes incluídos na amostra que apresentaram associação com o alto nível de conhecimento sobre o tratamento farmacológico com nível de significância menor que 0,20 na análise bivariada $(\mathrm{n}=130)$

\begin{tabular}{|c|c|c|c|}
\hline Características & Razão de chances & Intervalo de confiança $(95 \%)$ & p-valor* \\
\hline Sexo feminino & 2,64 & $1,08-6,51$ & 0,031 \\
\hline Idade $\leq 50$ anos & 3,15 & $1,45-6,92$ & 0,003 \\
\hline Chefe de família & 0,32 & $0,14-0,73$ & 0,005 \\
\hline $\begin{array}{l}\text { Escolaridade máxima ensino } \\
\text { fundamental completo }\end{array}$ & 0,26 & $0,11-0,61$ & 0,001 \\
\hline Exerce atividade remunerada & 2,04 & $0,81-5,18$ & 0,146 \\
\hline $\begin{array}{l}\text { Tempo de diagnóstico da doença renal } \\
\text { crônica } \geq 5 \text { anos }\end{array}$ & 2,44 & $1,13-5,29$ & 0,021 \\
\hline $\begin{array}{l}\text { Tempo de acompanhamento pelo } \\
\text { ambulatório de nefrologia } \leq 5 \text { anos }\end{array}$ & 0,57 & $0,27-1,22$ & 0,163 \\
\hline Declínio rápido do ritmo de filtração glomerular & 2,63 & $1,08-6,48$ & $\mathbf{0 , 0 3 1}$ \\
\hline Pressão arterial controlada & 2,37 & $0,95-5,95$ & 0,065 \\
\hline $\begin{array}{l}\text { Desconhece o risco de ser submetido à terapia de } \\
\text { substituição renal em caso de não utilização dos } \\
\text { medicamentos conforme prescrito }\end{array}$ & 0,55 & $0,24-1,25$ & 0,170 \\
\hline Número de medicamentos prescritos $\geq 4$ & 1,89 & $0,88-4,04$ & 0,107 \\
\hline $\begin{array}{l}\text { Utiliza algum medicamento pertencente aos } \\
\text { grupos H02 ou L04 da classificação }\end{array}$ & 3,04 & $1,36-6,82$ & 0,005 \\
\hline Anatomical Therapeutic Chemical & & & \\
\hline $\begin{array}{l}\text { Tempo médio de utilização dos } \\
\text { medicamentos } \leq 3 \text { anos }\end{array}$ & 0,39 & $0,18-0,84$ & 0,014 \\
\hline $\begin{array}{l}\text { Acredita já ter sofrido reação adversa a } \\
\text { algum medicamento prescrito }\end{array}$ & 6,14 & $2,53-15,16$ & $<0,001$ \\
\hline
\end{tabular}

*Teste do Qui-quadrado corrigido por Yates.

TABELA IV - Características dos pacientes incluídos na amostra que apresentaram associação estatisticamente significante com o alto nível de conhecimento sobre o tratamento farmacológico na análise multivariada $\left(\mathrm{n}=108^{1}\right)$

\begin{tabular}{lccc}
\hline Características & Razão de chances ajustada & Intervalo de confiança(95\%) & p-valor $^{2}$ \\
\hline Declínio rápido do ritmo de filtração glomerular & 3,33 & $1,24-8,96$ & $\mathbf{0 , 0 1 7}$ \\
Pressão arterial controlada & 4,87 & $1,60-14,77$ & $\mathbf{0 , 0 0 5}$ \\
Acredita já ter sofrido reação adversa a & 15,57 & $4,96-48,90$ & $<\mathbf{0 , 0 0 1}$ \\
algum medicamento prescrito & & & \\
\hline
\end{tabular}

\footnotetext{
${ }^{1}$ Vinte e dois pacientes não tinham dados disponíveis para todas as variáveis incluídas no modelo.

${ }^{2}$ Regressão logística múltipla.
} 
Hofmann, 2000; Silva, Schenkel, Mengue, 2000; Sano et al., 2002; Vasquez et al., 2003; Burge et al., 2005). O presente estudo, que teve por objetivo mensurar o nível de conhecimento sobre o tratamento farmacológico em pacientes com DRC e identificar os fatores que estivessem associados a este conhecimento, é parte de um outro trabalho, que teve por objetivo avaliar a adesão ao tratamento farmacológico e identificar os fatores associados a este comportamento (Moreira, 2005).

Foram encontrados na literatura outros dois estudos feitos no Brasil, nos últimos dez anos, que avaliaram o nível de conhecimento dos pacientes sobre os medicamentos prescritos. Estes trabalhos, utilizando instrumentos e escores semelhantes aos empregados na presente investigação, também foram realizados em ambulatórios de referência que atendiam aos pacientes através do Sistema Único de Saúde e que exerciam atividades de ensino. Entretanto as populações avaliadas nestes estudos diferem da descrita no presente trabalho principalmente pelas suas características clínicas e medicamentos em uso (Silva, Schenkel, Mengue, 2000; Ceccato et al., 2004). Já as investigações internacionais revisadas que avaliaram o nível de conhecimento dos pacientes sobre os medicamentos nos últimos dez anos foram realizadas em diferentes contextos sociais e clínicos, utilizando diferentes instrumentos de coleta e métodos de análise dos dados, o que dificulta a comparação dos resultados (Okuno et al., 1999; Vilke et al., 2000; Boonstra et al., 2003; Roche-Nagle et al., 2003; Ponnusankar et al., 2004; Burge et al., 2005; Dunning, Manias, 2005).

Na presente investigação foi observado que $77,7 \%$ dos pacientes sabiam dizer corretamente os nomes de todos os medicamentos que utilizavam. Este resultado é ligeiramente superior aos resultados de Silva, Schenkel e Mengue (2000) em que $69 \%$ dos entrevistados sabiam os nomes dos medicamentos enquanto Ceccato e colaboradores (2004) encontraram um índice de acerto de 72,5\%. Já os estudos internacionais encontraram resultados que variaram entre 2,5\% e 48\% (Okuno et al., 1999; Vilke et al., 2000; Boonstra et al., 2003). As doses dos medicamentos foram relatadas corretamente por $90 \%$ dos indivíduos. Os estudos nacionais relatam 81\% (Silva, Schenkel, Mengue, 2000) e 92,8\% (Ceccato et al., 2004). Trabalhos realizados em outros países encontraram taxas de acerto variando entre $24 \%$ e $91 \%$ (Okuno et al., 1999; Vilke et al., 2000; Boonstra et al., 2003; Roche-Nagle et al., 2003; Dunning, Manias, 2005). Foi observado ainda que $86,1 \%$ e $62,3 \%$ dos pacientes sabiam dizer corretamente as freqüências das administrações e os intervalos entre as doses, respectivamente. Silva, Schenkel e Mengue (2000) observaram que $69 \%$ dos entrevistados sabiam as freqüências das administrações, enquanto Ceccato e colaboradores (2004) encontraram um índice de acerto de
91,7\%. Estudos internacionais encontraram 39\% (Vilke et al., 2000) e 93\% (Dunning, Manias, 2005) de indivíduos que sabiam quando tomar seus medicamentos. Aproximadamente $42 \%$ dos pacientes incluídos na presente investigação não relataram corretamente as durações dos tratamentos, resultados semelhantes aos encontrados por Silva, Schenkel e Mengue (2000), 39\%, abaixo da freqüência encontrada por Boonstra e colaboradores (2003), 56\%, mas acima da encontrada por Ceccato e colaboradores (2004), que foi de $15,1 \%$. O desconhecimento dos nomes e dos regimes posológicos dos medicamentos prescritos pode causar erros graves no momento da aquisição ou da utilização dos mesmos. Estes erros podem levar à inefetividade do tratamento, que no caso da DRC favorece a progressão da doença e a necessidade precoce de TSR, e também à ocorrência de reações iatrogênicas potencialmente fatais.

Cerca de $25 \%$ dos pacientes cometeram algum erro ao descrever a indicação terapêutica dos medicamentos, resultado semelhante aos encontrados por Silva, Schenkel e Mengue (2000), 19\%, e Ceccato e colaboradores (2004), 28,8\%. A literatura internacional relata freqüências de desconhecimento da indicação variando entre 8\% e 65,7\% (Okuno et al., 1999; Boonstra et al., 2003; Roche-Nagle et al., 2003). Apenas $15,4 \%$ dos entrevistados citaram corretamente alguma reação adversa para todos os medicamentos que utilizavam, resultado semelhante ao encontrado por Silva, Schenkel e Mengue (2000), que foi de 16\%, mas discrepante de outros estudos encontrados na literatura, que relatam acertos entre $37 \% \mathrm{e}$ $61,1 \%$ para as respostas a perguntas semelhantes (Ceccato et al., 2004; Roche-Nagle et al., 2003; Dunning, Manias, 2005). O resultado do presente trabalho sugere que os pacientes têm pouca informação sobre as reações adversas que os medicamentos podem lhes causar, possivelmente porque os profissionais de saúde envolvidos na assistência são receosos quanto a fornecer estas informações e assim desencorajar os indivíduos a aderir ao tratamento.

No presente estudo o escore médio do nível de conhecimento sobre o tratamento farmacológico foi de 7,8 $\pm 1,7$ pontos, resultado semelhante à média de $7,7 \pm 1,5$ pontos encontrada por Ceccato e colaboradores (2004), que utilizaram uma escala semelhante de zero a dez pontos. Estudos internacionais, empregando escalas diferentes, encontraram escores médios que representavam entre $62 \%$ e $78 \%$ do escore máximo (Boonstra et al., 2003; Burge et al., 2005). Dez por cento dos pacientes apresentaram baixo nível de conhecimento sobre o tratamento farmacológico, enquanto $41,5 \%$ e 48,5\% alcançaram os níveis médio e alto, respectivamente. Este resultado preocupa, pois mais da metade dos pacientes não possui um nível de conhecimento que garanta a utilização correta e segura dos medicamentos em todas as circunstâncias. No estudo de Silva, Schenkel e Mengue 
(2000) estas prevalências foram de $9 \%, 57 \%$ e $34 \%$, respectivamente, enquanto no estudo de Ceccato e colaboradores (2004) cerca de $26 \%$ dos pacientes alcançaram menos de 7 pontos e foram considerados pacientes com nível insuficiente de conhecimento sobre os medicamentos.

$\mathrm{Na}$ análise multivariada foi observado que o declínio rápido do RFG foi uma característica que apresentou associação estatisticamente significante com o alto nível de conhecimento sobre o tratamento. Este resultado, aparentemente paradoxal, poderia ser explicado pelo fato de que indivíduos com maior taxa de declínio no RFG, o que significa uma progressão mais rápida da $\mathrm{DRC}$, geralmente recebem acompanhamento clínico mais intenso e este fato poderia influenciar aumentando o nível de conhecimento sobre os medicamentos.

A freqüência de pacientes com a pressão arterial controlada foi maior entre os que apresentaram alto nível de conhecimento sobre a utilização dos medicamentos do que entre aqueles que apresentaram os níveis baixo ou médio. $\mathrm{O}$ modelo de regressão logística múltipla estabeleceu que a diferença foi estatisticamente significante. Este resultado demonstrou que os indivíduos com maior nível de conhecimento sobre o tratamento farmacológico, apresentaram benefício clínico importante, pois o controle da pressão arterial é fundamental para a prevenção da progressão da DRC (National Kidney Foundation, 2002; Bregman, 2004).

No presente estudo foi observado que os pacientes com alto nível de conhecimento sobre os medicamentos possuíam uma chance aproximadamente 15 vezes maior de acreditar já ter sofrido reação adversa a algum dos medicamentos analisados, quando comparados àqueles com níveis de conhecimento baixo ou médio. Este resultado sugere que pacientes mais bem informados sobre o tratamento são capazes de identificar melhor os seus efeitos adversos. Entretanto, como os relatos não foram investigados é possível também que os indivíduos conhecedores das reações adversas estivessem atribuindo aos medicamentos sintomas de origem não farmacológica.

Os resultados deste estudo apontam para a necessidade de melhorias no processo de orientação aos pacientes sobre a DRC e o seu tratamento farmacológico, contemplando toda a equipe de profissionais envolvidos na assistência. Em um estudo de intervenção randomizado realizado na Índia os autores demonstraram melhorias importantes no nível de conhecimento dos pacientes que receberam aconselhamento quando comparados a pacientes que não receberam a intervenção (Ponnusankar et al., 2004). Intervenções baseadas na educação dos pacientes sobre a doença e o tratamento também têm se mostrado eficazes na promoção da adesão ao tratamento (Márquez Contreras et al., 1998; Morrison, Wertheimer, 2001; Peterson, Takiya, Finley, 2003).
Entre as principais limitações do estudo estão as anotações incompletas e ilegíveis nos prontuários dos pacientes, que prejudicaram a coleta dos dados. O delineamento transversal adotado não permite que sejam definidas relações de causalidade entre o nível de conhecimento sobre os medicamentos e as características a ele associados. A principal limitação para a associação entre o conhecimento sobre o tratamento e os resultados terapêuticos está na relação inexata entre adesão e os efeitos do tratamento farmacológico (Gordis, 1979). Na doença renal crônica o RFG pode diminuir rapidamente mesmo se os medicamentos forem tomados corretamente, pois existem doenças glomerulares que não respondem ao tratamento. A proteinúria pode aumentar mesmo com a adequada adesão e a hipertensão arterial, em casos como o da nefrite lúpica e o da síndrome nefrótica descompensada, pode ser difícil de ser controlada mesmo com o uso correto dos medicamentos.

Estudos observacionais prospectivos e com maior número de pacientes são necessários para estabelecer as causas do inadequado conhecimento sobre os medicamentos prescritos e estudos de intervenção randomizados para avaliar a efetividade de intervenções direcionadas para a melhoria do nível de conhecimento sobre o tratamento farmacológico dos pacientes.

\section{CONCLUSÕES}

Os resultados do presente estudo permitiram concluir que a maioria dos pacientes com DRC atendidos pelo ambulatório de nefrologia do HUWC/UFC possuía nível de conhecimento baixo ou médio sobre o tratamento farmacológico. Os fatores que estiveram associados com o alto nível de conhecimento, de forma independente, foram: declínio rápido do $\mathrm{RFG}$, controle da pressão arterial e crença do paciente sobre já ter sofrido reação adversa a algum medicamento prescrito.

\section{ABSTRACT \\ Medication knowledge in patients with chronic kidney disease}

The suitable patients' medication knowledge is a essential factor for the medication compliance. The level of medication knowledge in patients with chronic kidney disease (CKD) and the medication knowledge associated factors were assessed in a cross-sectional study performed with 130 subjects from a nephrology outpatient university clinic, continuously taking some self-administered drug from the C, H02 or L04 groups of the Anatomical Therapeutic Chemical classification system and who were not on a kidney replacement therapy. 
The level of medication knowledge was measured through a researcher-administered questionnaire and a score ranking from zero to ten points. Three levels of knowledge were defined: low (less than six points), moderate (six to eight points) and high (more than 8 points). The patients' medication knowledge average score was $7.8 \pm 1.7$ points and $51.5 \%$ of the patients showed low or moderate level of knowledge. The factors associated to the high level of medication knowledge were: fast decline of the glomerular filtration rate, control of the blood pressure and the patients' belief that they suffered adverse drug reaction to any of the prescribed medicines. Most patients had insufficient knowledge for a safe and effective use of prescribed medicines, so a better patient counseling is necessary.

UNITERMS: Drugs/continuous use. Chronic kidney failure/pharmacological treatment. Drugs/safety utilization.

\section{AGRADECIMENTOS}

Os autores gostariam de agradecer aos pacientes, médicos, enfermeiras, estudantes e demais funcionários do HUWC/UFC, ao CIM/GPUIM/UFC e à Farm. Ana Cláudia de Brito Passos pela contribuição para a realização deste trabalho.

\section{REFERÊNCIAS BIBLIOGRÁFICAS}

ALVES, M.A.R. Diagnóstico de doença renal crônica: avaliação de proteinúria e sedimento urinário. J. Bras. Nefrol., v.26, n.3 (supl.1), p.6-8, 2004.

AMERICAN DIABETES ASSOCIATION. Clinical practice recommendations. Diabetes Care, v.29, suppl.1, p.S4S85, 2006.

BOONSTRA, E.; LINDBAEK, M.; NGOME, E.; TSHUKUDU, K.; FUGELLI, P. Labelling and patient knowledge of dispensed drugs as quality indicators in primary care in Botswana. Qual. Saf. Health Care, v.12, n.3, p.168-175, 2003.

BREGMAN, R. Prevenção da progressão da doença renal crônica (DRC). J. Bras. Nefrol., v.26, n.3 (supl.1), p.1114, 2004.

BUNZEL, B.; LAEDERACH-HOFMANN, K. Solid organ transplantation: are there predictors for posttransplant noncompliance? A literature overview. Transplantation, v.70, n.5, p.711-716, 2000.
BURGE, S.; WHITE, D.; BAJOREK, E.; BAZALDUA, O.; TREVINO, J.; ALBRIGHT, T.; WRIGHT, F.; CIGARROA, L. Correlates of medication knowledge and adherence: findings from the residency research network of South Texas. Fam. Med., v.37, n.10, p.712-718, 2005.

CECCATO, M.G.B.; ACURCIO, F.A.; BONOLO, P.F.; ROCHA, G.M.; GUIMARÃES, M.D.C. Compreensão de informações relativas ao tratamento anti-retroviral entre indivíduos infectados pelo HIV. Cad. Saúde Pública, v.20, n.5, p.1388-1397, 2004.

CHOBANIAN, A.V.; BAKRIS, G.L.; BLACK , H.R.; CUSHMAN, W.C.; GREEN, L.A.; IZZO JUNIOR, J.L.; JONES, D.W.; MATERSON, B.J.; OPARIL, S.; WRIGHT JUNIOR, J.T.; ROCCELLA, E.J.; National Heart, Lung and Blood Institute Joint National Committee on Prevention, Detection, Evaluation and Treatment of High Blood Pressure; National High Blood Pressure Education Program Coordinating Committee. The seventh report of the Joint National Committee on Prevention, Detection, Evaluation, and Treatment of High Blood Pressure: the JNC 7 report. JAMA, v.289, n.19, p.25602572, 2003.

CRAMER, J.A. Effect of partial compliance on cardiovascular medication effectiveness. Heart, v.88, n.2, p.203-206, 2002.

DUNNING, T.; MANIAS, E. Medication knowledge and selfmanagement by people with type 2 diabetes. Aust. J. Adv. Nurs., v.23, n.1, p.7-14, 2005.

FARLEY, D. FDA's Rx for better medication information. FDA Consum., v.29, n.9, p.5-10, 1995.

FRASSINETTI FERNANDES, P.; ELLIS, P.A.; RODERICK, P.J.; CAIRNS, H.S.; HICKS, J.A.; CAMERON, J.S. Causes of end-stage renal failure in black patients starting renal replacement therapy. Am. J. Kidney Dis., v.36, n.2, p.301-309, 2000.

GORDIS, L. Conceptual and methodologic problems in measuring patient compliance. In: HAYNES, R.B.; TAYLOR, D.W.; SACKETT, D.L., (Eds.). Compliance in health care. Baltimore: Johns Hopkins University, 1979. cap.3, p.23-45.

GRANT, R.W.; DEVITA, N.G.; SINGER, D.E.; MEIGS, J.B. Polypharmacy and medication adherencein patients with type 2 diabetes. Diabetes Care, v.26, n.5, p.1408-1412, 2003. 
KILBOURNE, A.M.; GOOD, C.B.; SEREIKA, S.M.; JUSTICE, A.C.; FINE, M.J. Algorithm for assessing patients' adherence to oral hypoglycemic medication. Am. J. Health. Syst. Pharm., v.62, n.2, p.198-204, 2005.

KUO, Y.F.; RAJI, M.A.; MARKIDES, K.S.; RAY, L.A.; ESPINO, D.V.; GOODWIN, J.S. Inconsistent use of diabetes medications, diabetes complications, and mortality in older Mexican Americans over a 7-year period. Diabetes Care, v.26, n.11, p.3054-3060, 2003.

LEE, J.Y, KUSEK, J.W; GREENE, P.G.; BERNHARD, S.; NORRIS, K.; SMITH, D.; WILKENING, B.; WRIGHT JUNIOR, J.T. Assessing medication adherence by pill count and electronic monitoring in the African American Study of Kidney Disease and Hypertension (AASK) Pilot Study. Am. J. Hypertens., v.9, n.8, p.719-725, 1996.

LEITE, S.N.; VASCONCELLOS, M.P.C. Adesão à terapêutica medicamentosa: elementos para a discussão de conceitos e pressupostos adotados na literatura. Ciência e Saúde Coletiva, v.8, n.3, p.775-782, 2003.

MÁRQUEZCONTRERAS, E.; CASADOMARTÍNEZ, J.J.; RAMOS PÉREZ, J.; SÁENZ SOUBRIER, S.; MORENO GARCÍA, J.P.; CELOTTI GÓMEZ, B.; GASCÓN VIVÓ, J.; MARTÍN DE PABLOS, J.L. Ensayo sobre la eficacia de los programas de educación para la salud en el cumplimiento terapéutico de la hipertensión arterial. Aten. Primaria, v.21, n.4, p.199-204, 1998.

MOREIRA, L.B. Adesão ao tratamento farmacológico em doentes renais crônicos atendidos pelo ambulatório do Hospital Universitário Walter Cantídio. Fortaleza, 2005. 198p. [Dissertação de Mestrado. Faculdade de Farmácia, Odontologia eEnfermagem. UniversidadeFederal do Ceará].

MORRISON,A.; WERTHEIMER, A.I. Evaluation of studies investigating the effectiveness of pharmacists' clinical services. Am. J. Health Syst. Pharm., v.58, n.7, p.569577, 2001.

NATIONAL KIDNEY FOUNDATION. K/DOQI clinical practice guidelines for chronic kidney disease: evaluation, classification, and stratification. Am. J. Kidney Dis., v.39, n.2 (suppl.1), p.S1-S266, 2002.

OKUNO, J.; YANAGI, H.; TOMURA, S.; OKA, M.; HARA, S.; HIRANO, C.; TSUCHIYA, S. Compliance and medication knowledge among elderly Japanese homecare recipients. Eur. J. Clin. Pharmacol., v.55, n.2, p.145149, 1999.
PARFITT, K., (Ed.). Martindale: the complete drug reference. 32. ed. London: Pharmaceutical Press, 1999. 2315p.

PECOITS-FILHO, R. Diagnóstico de doença renal crônica: avaliação da função renal. J. Bras. Nefrol., v.26, n.3, supl.1, p.4-5, 2004.

PETERSON,A.M.; TAKIYA, L.; FINLEY, R. Meta-analysis of trials of interventions to improve medication adherence. Am. J. Health Syst. Pharm., v.60, n.7, p.657-665, 2003.

PONNUSANKAR, S.; SURULIVELRAJAN, M.; ANANDAMOORTHY, N.; SURESH, B. Assessment of impact of medication counseling on patients' medication knowledge and compliance in an outpatient clinic in South India. Patient Educ. Couns, v.54, n.1, p.55-60, 2004

ROCHE-NAGLE, G.; CHAMBERS, F.; NANRA, J.; BOUCHIER-HAYES, D.; YOUNG, S. Evaluation of patient knowledge regarding oral anticoagulants. Ir. Med. J., v.96, n.7, p.211-213, 2003.

ROMÃO JÚNIOR, J.E. Doença renal crônica: definição, epidemiologia e classificação. J. Bras. Nefrol., v.26, n.3, supl.1, p.1-3, 2004

RYBACKI, J.J. Improving cardiovascular health in postmenopausal women by addressing medication adherence issues. J. Am. Pharm. Assoc., v.42, n.1, p.63-71.

SANO, P.Y.; MASOTTI, R.R.; DOS SANTOS, A.A.; CORDEIRO, J.A. Avaliação do nível de compreensão da prescrição pediátrica. J. Pediatr., v.78, n.2, p.140-145, 2002.

SARQUIS, L.M.M.; DELL'ACQUA, M.C.Q.; GALLANI, M.C.B.J.; MOREIRA, R.M.; BOCCHI, S.C.M.; TASE, T.H.; PIERIN, A.M.G. A adesão ao tratamento na hipertensão arterial: análise da produção científica. Rev. Esc. Enf., v.32, n.4, p.335-353, 1998.

SILVA, T. Caracterização e análise do nivel de informação sobre medicamentos prescritos a pacientes ambulatoriais do Hospital de Clínicas de Porto Alegre. Porto Alegre, 1999. p.167. [Dissertação de Mestrado. Faculdade de Farmácia. Universidade Federal do Rio Grande do Sul].

SILVA, T.; SCHENKEL, E.P.; MENGUE, S.S. Nível de informação a respeito de medicamentos prescritos a pacientes ambulatoriais de Hospital Universitário. Cad. Saúde Pública, v.16, n.2, p.449-455, 2000. 
SOCIEDADE BRASILEIRA DE NEFROLOGIA. Campanha de prevenção de doença renal: por que a campanha? Informações sobre doença renal (estatísticas brasileiras, sintomatologia). Disponível em: $<$ http:// www.sbn.org.br/previna.htm>. Acesso em: 03 mai. 2006.

STRELEC, M.A.; PIERIN, A.M.; MION JÚNIOR, D. The influence of patient's consciousness regarding high blood pressure and patient's attitude in face of disease controlling medicine intake. Arq. Bras. Cardiol., v.81, n.4, p.349-354, 2003.

TEIXEIRA, J.J.V.; LEFÈVRE, F.; CASTRO, L.L.C.; SPÍNOLA, A.W.P. Drug compliance and the elderly: who is publishing, where, and when? Cad. Saúde Pública, v.16, n.1, p.139-144, 2000.
VASQUEZ, E.M.; TANZI, M.; BENEDETTI, E.; POLLAK, R. Medication compliance after kidney transplantation. Am. J. Health Syst. Pharm., v.60, n.3, p.266-269, 2003.

VILKE, G.M.; MARINO, A.; ISKANDER, J.; CHAN, T.C. Emergency department patient knowledge of medications. J. Emerg. Med., v.19, n.4, p.327-330, 2000.

WORLD HEALTH ORGANIZATION. Adherence to longterm therapies: evidence for action. Geneva, 2003. 198p.

WORLD HEALTH ORGANIZATION. ATC index with DDD's 2005.Disponível em: <http://www.whocc.no/ atcddd/>. Acesso em: 25 jun. 2005.

Recebido para publicação em 06 de agosto de 2007 Aceito para publicação em 29 de janeiro de 2008 